\title{
Research on Weak Fault Extraction Method for Alleviating the Mode Mixing of LMD
}

\author{
Lin Zhang ${ }^{1,2, *}$, Zhijian Wang ${ }^{3, *}$ and Long Quan ${ }^{1,2}$ \\ 1 College of Mechanical Engineering, Taiyuan University of Technology, Taiyuan 030024, China; \\ quanlong@tyut.edu.cn \\ 2 Key Laboratory of Advanced Transducers and Intelligent Control System, Ministry of Education, \\ Taiyuan 030024, China \\ 3 College of Mechanical and Power Engineering, The North University of China, Taiyuan 030051, China \\ * Correspondence: zhanglin@tyut.edu.cn (L.Z.); wangzhijian1013@163.com (Z.W.)
}

Received: 10 April 2018; Accepted: 16 May 2018; Published: 21 May 2018

\begin{abstract}
Compared with the strong background noise, the energy entropy of early fault signals of bearings are weak under actual working conditions. Therefore, extracting the bearings' early fault features has always been a major difficulty in fault diagnosis of rotating machinery. Based on the above problems, the masking method is introduced into the Local Mean Decomposition (LMD) decomposition process, and a weak fault extraction method based on LMD and mask signal (MS) is proposed. Due to the mode mixing of the product function (PF) components decomposed by LMD in the noisy background, it is difficult to distinguish the authenticity of the fault frequency. Therefore, the MS method is introduced to deal with the PF components that are decomposed by the LMD and have strong correlation with the original signal, so as to suppress the modal aliasing phenomenon and extract the fault frequencies. In this paper, the actual fault signal of the rolling bearing is analyzed. By combining the MS method with the LMD method, the fault signal mixed with the noise is processed. The kurtosis value at the fault frequency is increased by eight-fold, and the signal-to-noise ratio (SNR) is increased by $19.1 \%$. The fault signal is successfully extracted by the proposed composite method.
\end{abstract}

Keywords: strong noise; local mean decomposition; fault diagnosis; product function

\section{Introduction}

Bearings are crucial parts of rotating machinery, and bearing wear is inevitable [1,2]. The early signs of bearing wear are very weak, and the signals are difficult to find in the strong noise background [3-5]. Bearing faults will occur if corresponding measures have not been taken, which can even lead to serious accidents, causing economic losses and personnel casualties, so the early fault signal extraction of bearings has always been highly valued [6-8]. The faults of rolling bearings can be mainly summarized as inner ring wear, outer ring wear and rolling body pitting, and periodic pulse signals will be produced when these faults occur. Due to the noise interference, the early fault signal extraction is always the most important and difficult part of fault diagnosis [9,10]. The existing fault diagnosis methods include traditional Fourier transform, wavelet decomposition and empirical mode decomposition (EMD).

Dragomiretskiy et al. proposed the variational modal decomposition (VMD) method in 2014 [11]. VMD converts the decomposition of the signal into a variational constraint problem. A non-recursive solution is used to avoid the mode mixing which exists in traditional decomposition methods such as empirical mode decomposition (EMD). VMD possesses good noise robustness and high computational efficiency. Wang [12] proposed a VMD method based on particle swarm optimization, which uses the 
minimum mean envelope entropy to optimize the parameters of VMD and achieved good results in the fault diagnosis of rotating machinery. Yang [13] proposed a method which mixes Ensemble Empirical Mode Decomposition (EEMD), sample entropy and Singular Value Decomposition (SVD) in acoustic signal fault diagnosis, where sample entropy and SVD are indicators of periodic and irregular signals respectively. This adaptive method has good robustness and it is suitable for processing non-stationary and non-linear signals.

In 2005, on the basis of EMD, Smith proposed a time-frequency analysis method call local mean decomposition (LMD), whose essence is to divide the signal by frequency into different product function (PF) components. Each PF component is an envelope signal multiplied by a pure FM signal. By analyzing PF components after the decomposition in the frequency domain, the complete time-frequency distribution can be obtained [14-16].

However, in comparison to EMD some disadvantages of LMD are also obvious. Thus, although the mode mixing of LMD has been reduced, the mode mixing is still serious. The mode mixing results in the mixing of time-frequency distribution and the generation of promiscuous PF components, so the required time-frequency information $[17,18]$ can't be extracted from them.

In order to solve the mode mixing of LMD, many scholars have proposed different methods in succession. Among these methods, Mask Signal (MS) has attracted much attention due to its high computational efficiency and strong post-processing ability, but so far, MS has not been applied to solve the mode mixing issues of LMD [19-21].

Based on the above reasons, this paper introduces MS into LMD, and finds that MS has a certain noise reduction ability, the influence of noise in the PF component has been reduced by MS. The frequency energy mean parameter is the basis of the refinement of the fault frequency band [22]. In the analysis of weak simulation signal and the fault signals of the rolling bearings, the mode mixing of LMD has been successfully weakened, and the fault features have been extracted. The validity of the method is verified by calculating the signal-to-noise ratio and kurtosis ratio before and after the signal processing. In theory and practice, a new diagnostic method for fault feature extraction is provided.

\section{Background and New Method}

\subsection{LMD Method}

For an original signal $x(t)$, the LMD decomposition steps are as follows:

(1) According to all the local extreme point $n_{i}$, all the local extreme mean values $m_{i}$ and the envelope estimate values $a_{i}$ are obtained.

The local mean function $m_{11}(t)$ and the envelope function $a_{11}(t)$ are obtained by the sliding average method.

(2) From the local mean function of the original signal $x(t)$, the function $h_{11}(t)$ is obtained, and then it is demodulated to the function $s_{11}(t)$ :

$$
\begin{gathered}
h_{11}(t)=x(t)-m_{11}(t) \\
s_{11}(t)=\frac{h_{11}(t)}{a_{11}(t)}
\end{gathered}
$$

$s_{11}(t)$ is used as a new function to repeat the above steps to get $s_{12}(t)$ until $s_{1 n}(t)$ is a pure FM function, that is $\lim _{n \rightarrow \infty} a_{1 n}(t)=1$.

(3) The envelope signal is obtained:

$$
a_{1}(t)=a_{11}(t) a_{12}(t) \cdots a_{1 n}(t)=\prod_{p=1}^{n} a_{1 p}(t)
$$


(4) The first PF component is obtained:

$$
P F_{1}(t)=a_{1}(t) s_{1 n}(t)
$$

(5) After $P F_{1}(t)$ is separated from $x(t)$ and $u_{1}(t)$ is obtained, $u_{1}(t)$ repeats the above calculation as a new original signal to obtain $u_{2}(t)$ until $u_{\mathrm{q}}(t)$ is a monotone function stop iteration:

$$
u_{1}(t)=x(t)-P F_{1}(t)
$$

(6) The original signal is decomposed into:

$$
x(t)=\sum_{i=1}^{q} P F_{i}(t)+u_{q}(t)
$$

\subsection{MS Method}

Deering [23] proposed the mask signal method to alleviate the mode mixing of EMD. The specific way is to determine the amplitude and the frequency of $s(t)$ according to the EMD's Hilbert envelope amplitude and instantaneous frequency. Good results have been obtained, but less attention has been paid to the mask signal method in alleviating the mode mixing of LMD.

The basic principle of MS is to reduce the accumulated error of smooth processing by adding and subtracting the average, weaken the mode mixing and noise, highlight the peak value near the mean instantaneous frequency. Finding the proper mask signal $s(t)$ is the key of MS:

(1) Suppose $t$ is time, $x(t)$ is the original signal, $\tau$ is the integral variable. We perform a Hilbert transform on the original signal $x(t)$ to get $y(t)$, and transform it to $z(t)$ :

$$
\begin{gathered}
y(t)=\frac{1}{\pi} \int_{-\infty}^{+\infty} \frac{x(t)}{\tau-t} d \tau \\
z(t)=x(t)+j y(t)=a_{i}(t) e^{j \phi_{i}(t)}
\end{gathered}
$$

(2) The instantaneous frequency is obtained according to the instantaneous phases of the amplitude function $a_{i}(t)$ and the phase function $\varphi_{i}(t)$ :

$$
f_{i}(t)=\frac{1}{2 \pi} \omega_{i}(t)=\frac{1}{2 \pi} \frac{d \phi_{i}(t)}{d t}
$$

Equation (7) can be calculated by the mean of the energy mean method:

$$
\bar{f}=\frac{\sum_{i}^{k} a_{1}(i) f_{1}^{2}(i)}{\sum_{i}^{k} a_{1}(i) f_{1}(i)}
$$

The average amplitude and the average instantaneous frequency are different when MS is used for different signal processing. In face of $x(t), a(t)$ is the envelope amplitude of $x(t), f_{1}(t)$ is the instantaneous frequency of $x(t)$, so the mask signal is determined as:

$$
s(t)=a_{0} \sin (2 \pi \bar{f} t)
$$

According to the rule of thumb, $a_{0}$ often takes 1.6 times of the average amplitude of the signal component.

(3) Create a mask signal $s(t)$, let:

$$
x_{+}(t)=x(t)-s(t)
$$




$$
x_{-}(t)=x(t)-s(t)
$$

(4) The original signal can be obtained from the combination of $x_{+}(t)$ and $x_{-}(t)$ :

$$
x(t)=\frac{x_{+}(t)+x_{-}(t)}{2}
$$

The performance of MS is verified by an example of the simulation signal in Equation (15). The simulation signal is shown in Figure 1. The frequencies of a, b and c are $40 \mathrm{~Hz}, 80 \mathrm{~Hz}$ and $130 \mathrm{~Hz}$, respectively:

$$
x(t)=\sin (80 \pi t)+0.8 \sin (160 \pi t)+\cos (260 \pi t)+1.5 \text { noise }(t)
$$
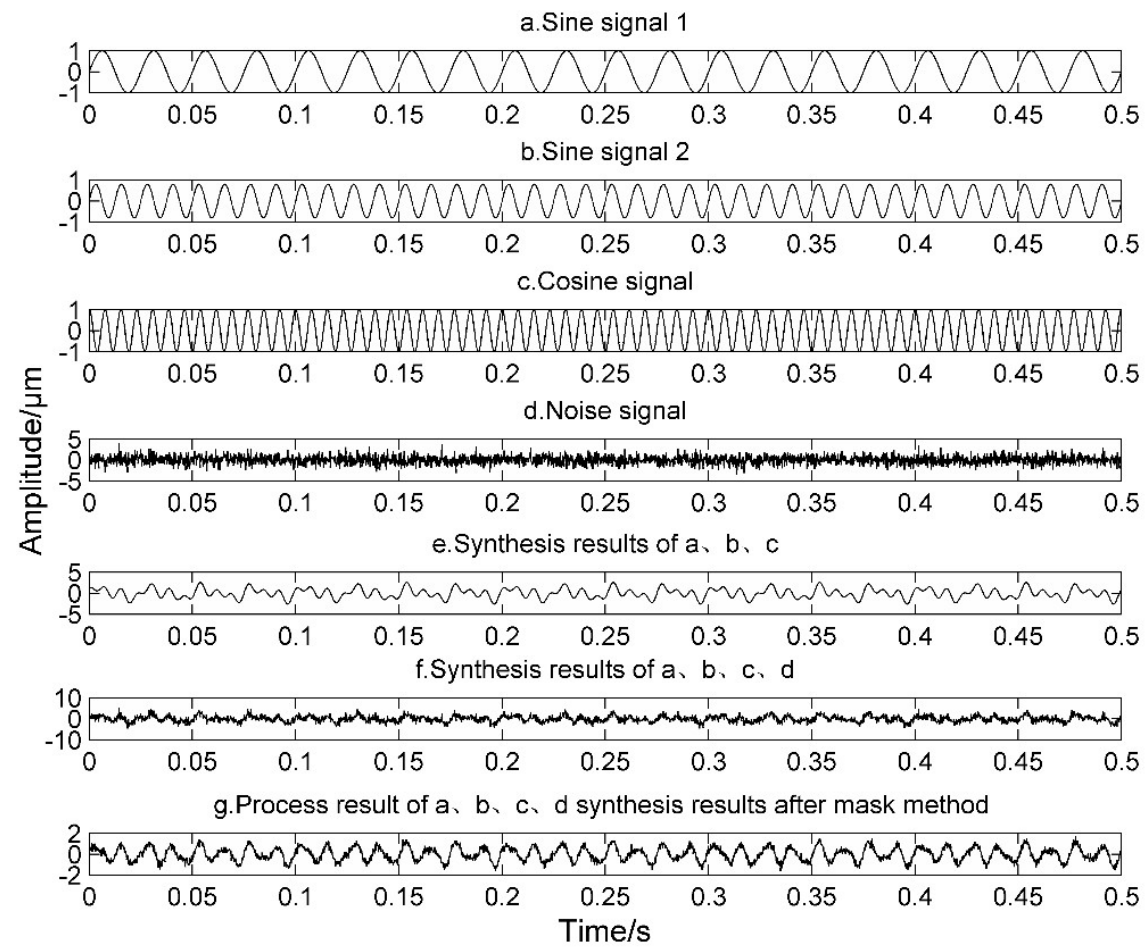

Figure 1. Simulation signal.

As can be seen from the results of e, $\mathrm{f}$, and $\mathrm{g}$ in Figure 1, MS has a certain noise reduction capability, and the waveform in the $g$ diagram has also changed. From the comparison results of the frequency domain diagram of $\mathrm{f}$ and $\mathrm{g}$ in Figure 2, the peak values of the $40 \mathrm{~Hz}$ and $130 \mathrm{~Hz}$ signals after the masking process are significantly weaker than those of the untreated signal. The peak value of $80 \mathrm{~Hz}$ is clearly prominent. This is due to the fact that the relevant parameters of the mask signals of MS are instantaneous amplitude and instantaneous frequencies. The mean instantaneous frequency of the simulation signal is about $83 \mathrm{~Hz}$. After masking, the peaks at $40 \mathrm{~Hz}$ and $130 \mathrm{~Hz}$ are weakened. 

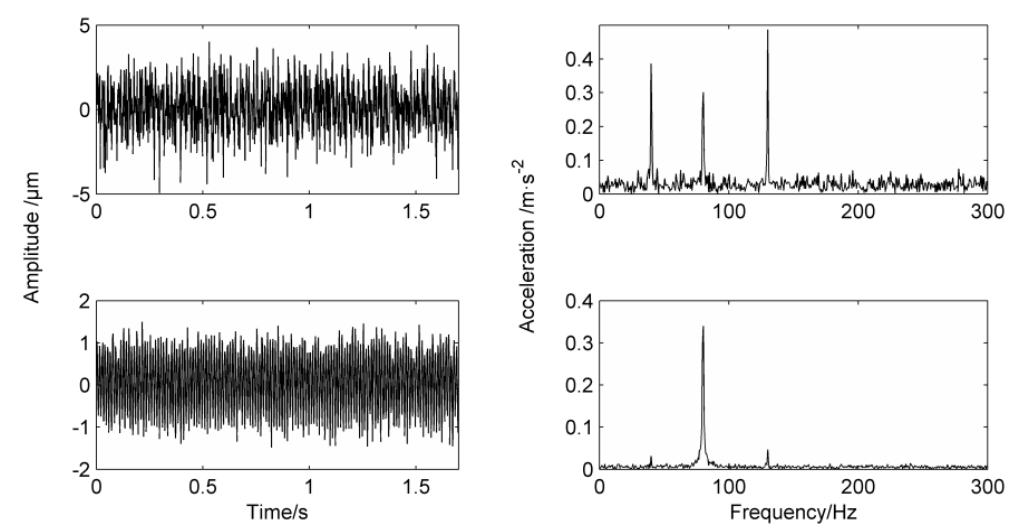

Figure 2. Simulation signal frequency domain of $\mathrm{f}$ and $\mathrm{g}$.

\section{The Basic Principle of LMD-MS}

Nakayama and others put forward a method for improving the performance of the prediction task by mask signal [24], which can be improved appropriately when applied to LMD. Since LMD adopts an envelope based on extreme points, the envelope estimation errors will be amplified after multiple decomposition, and the mode mixing will appear. The MS method takes the mean method after adding and subtracting, which can decrease the accumulated errors resulted from the multiple smooth processing to alleviate the mode mixing and the noise.

Taking the first $\mathrm{PF}$ component $\mathrm{PF}_{1}$ decomposed by LMD as an example, the decomposed $\mathrm{PF}_{1}$ is used as the original signal, and the mask signal is determined, the $\mathrm{PF}_{1}$ is decomposed into $\mathrm{PF}^{+}$and $\mathrm{PF}^{-}$, redefine $\mathrm{PF}_{1}$ :

$$
\mathrm{PF}_{1}=\frac{\mathrm{PF}^{+}+\mathrm{PF}^{-}}{2}
$$

When the LMD-MS method deals with a single fault bearing signal, the PF component who has the highest relativity with the original signal can be selected as the target for mask processing.

In the fault signal, the fault impact will appear periodically, so in this frequency band, the frequency energy means will be higher than those of other frequency bands. Figure 3 is the frequency energy mean diagram of the simulation signal in Equation (15).

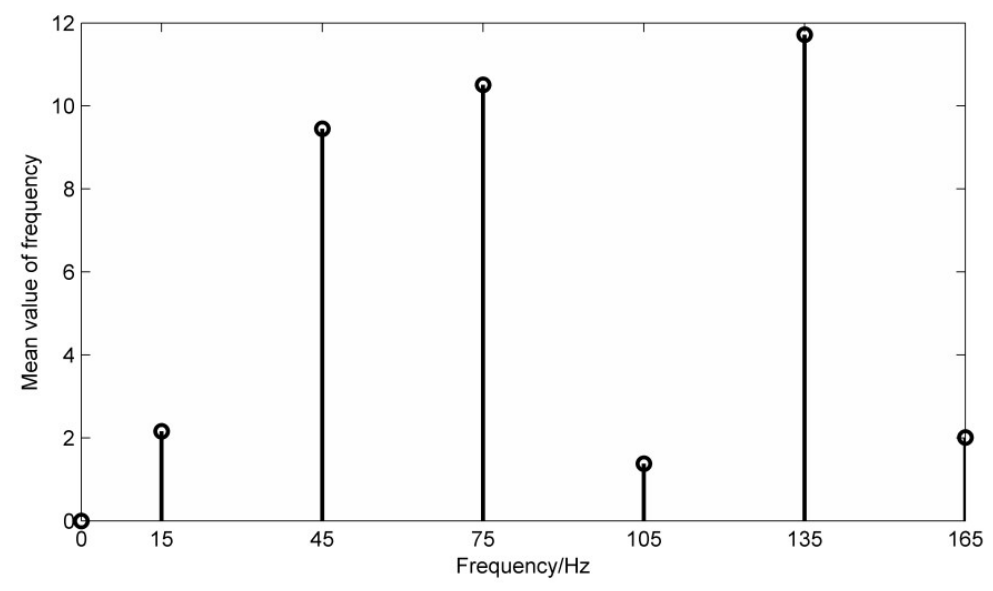

Figure 3. Simulation signal frequency energy mean distribution.

In Figure 3, taking about $15 \mathrm{~Hz}$ of each point as the frequency energy mean, it can be obtained that $45 \mathrm{~Hz}, 75 \mathrm{~Hz}$ and $135 \mathrm{~Hz}$ are at higher frequency mean energy. When the mean instantaneous frequency of the masking signal is adjusted to $40 \mathrm{~Hz}$ and $130 \mathrm{~Hz}$, the results are shown in Figure 4. 

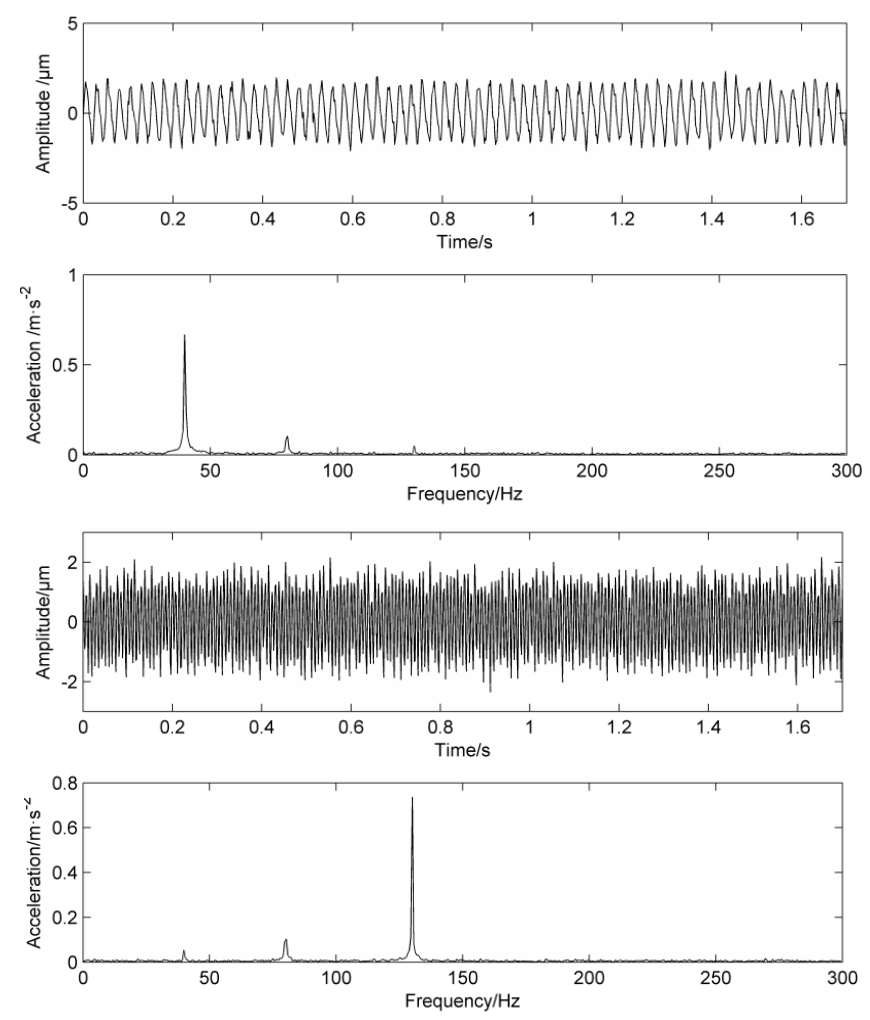

Figure 4. The time-frequency domain of the simulated signal with mean instantaneous frequency of $40 \mathrm{~Hz}$ and $130 \mathrm{~Hz}$.

When the mean instantaneous frequency is $80 \mathrm{~Hz}$, the time-frequency domain diagram is basically the same as Figure 2, and from the results of Figures 2 and 4, we can see that the selection of the mask signal is the key point. When using the mask method to process the signal, we can choose the frequency with the highest frequency energy as the basis for selecting the mask signal. The flow chart of the fault diagnosis method based on MS and LMD is as follows in Figure 5:

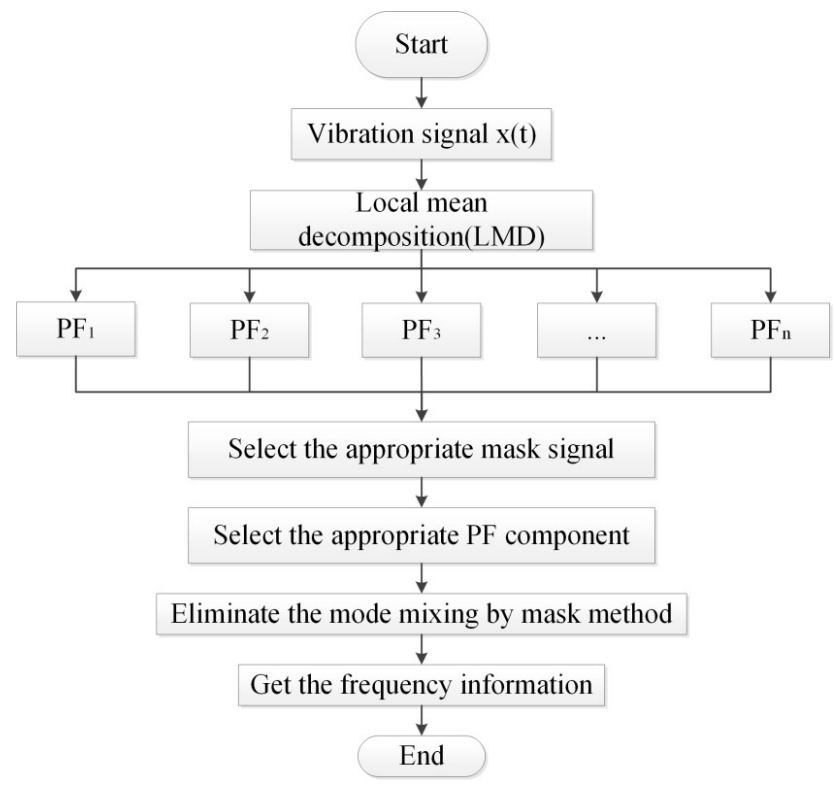

Figure 5. Flow chart of LMD-MS. 


\section{Simulation Signal Analysis}

In order to verify the mode mixing phenomenon of LMD in noise, the modulated simulation signal in Equation (17) is adopted, the corresponding frequencies are $40 \mathrm{~Hz}, 120 \mathrm{~Hz}$ and $260 \mathrm{~Hz}$ respectively, and the corresponding time-domain waveform of simulation signal is shown in Figure 6.

$$
x(t)=[1+0.8 \cos (240 \pi t)+\cos (520 \pi t)] \cdot \sin (80 \pi t)+\text { noise }(t)
$$

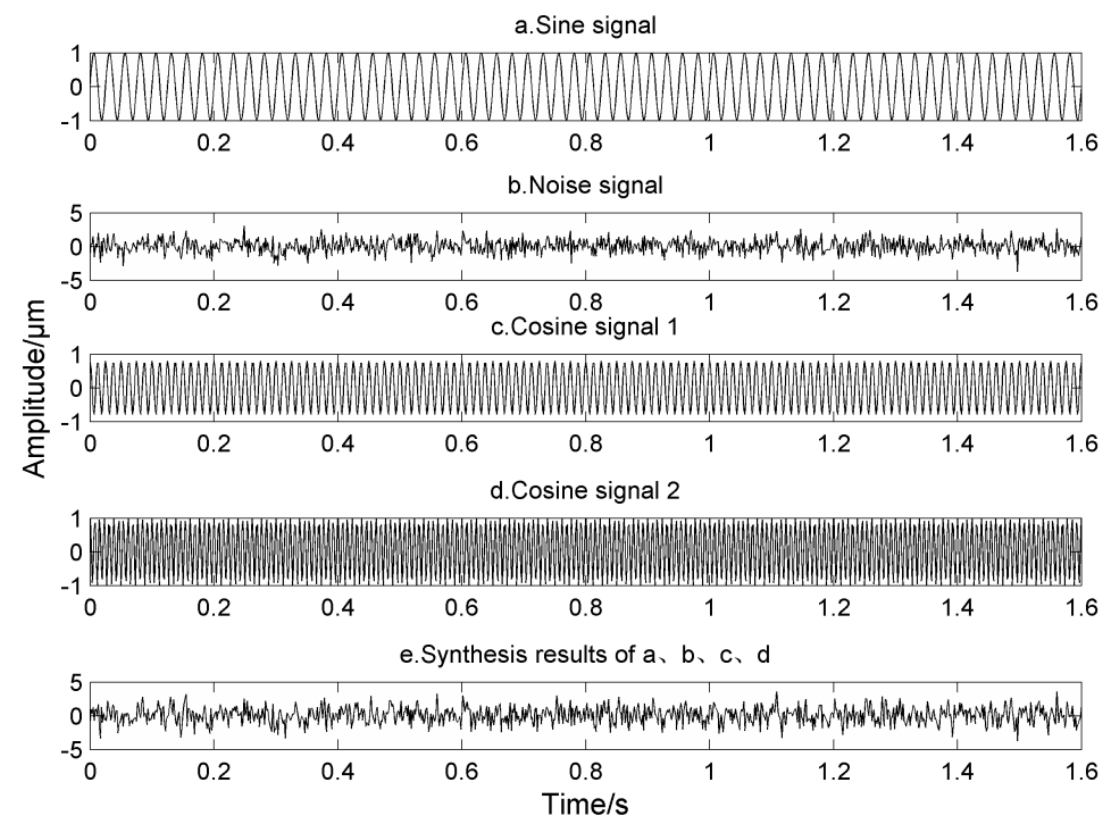

Figure 6. Simulation signal.

In Figure 6, from top to bottom, the signals are a sine signal, the noise signal, two cosine signals and the time domain diagrams of the synthesized simulation signal, where the two cosine functions are modulated by a sine function.

The frequency domain diagram of the PF component that is directly processed by LMD decomposition without a mask signal method is shown in Figure 7. The first layer is the time-frequency domain of $\mathrm{PF}_{1}$, it can be clearly seen from the frequency domain that there are high peaks at $120 \mathrm{~Hz}$, $260 \mathrm{~Hz}$ and a lower peak at $40 \mathrm{~Hz}$. The second layer is the time-frequency domain of $\mathrm{PF}_{2}$, and there are significant peaks at $40 \mathrm{~Hz}$ and $120 \mathrm{~Hz}$. At the same time, there are many false frequencies in the spectrum due to the noise interference. The third layer is the time-frequency domain of $\mathrm{PF}_{3}$, the frequency graph only has a higher peak at $40 \mathrm{~Hz}$, the fourth layer and the fifth layer is false components which can be dropped.

From the comparison of the first three layers of the PF components, it can be concluded that $120 \mathrm{~Hz}$ and $260 \mathrm{~Hz}$ belong to high-frequency components, $40 \mathrm{~Hz}$ belongs to low-frequency components, but $120 \mathrm{~Hz}$ and $260 \mathrm{~Hz}$ appear in the spectrum of $\mathrm{PF}_{1}$ at the same time. $40 \mathrm{~Hz}$ and $120 \mathrm{~Hz}$ appear in the spectrum of $\mathrm{PF}_{2}$ at the same time, which proves that mode mixing occurs.

The mean frequency energy parameters of the simulation signal are calculated and the required frequency information is filtered. Regard the frequency value before and after $5 \mathrm{~Hz}$ as the frequency energy mean length, which is shown in Figure 8.

The results obtained from Figure 8 show that the mean frequencies of $40 \mathrm{~Hz}, 120 \mathrm{~Hz}$ and $260 \mathrm{~Hz}$ are the highest, and the mask signal is selected according to the three frequency values. 

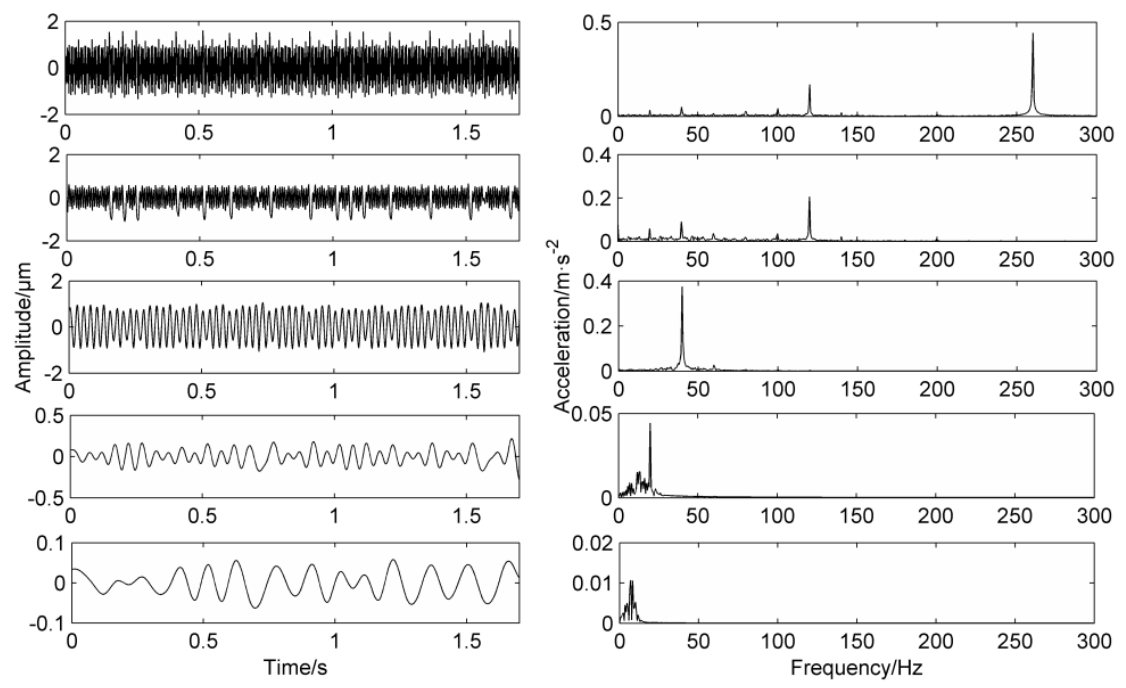

Figure 7. Simulation signal's decomposition results using LMD.

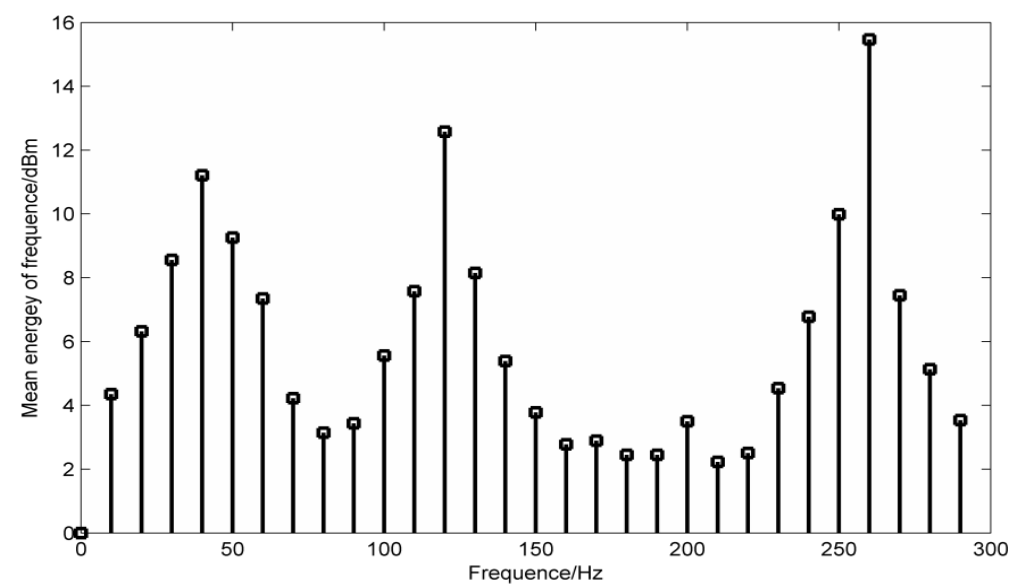

Figure 8. Simulation signal frequency energy mean distribution.

When the mean instantaneous frequency of the mask signal is selected as $40 \mathrm{~Hz}$, the corresponding results can be obtained for $\mathrm{PF}_{1}, \mathrm{PF}_{2}$ and $\mathrm{PF}_{3}$, but the results obtained for $\mathrm{PF}_{3}$ are the best. When the mean instantaneous frequency of the mask signal is $260 \mathrm{~Hz}$, the treatment of $\mathrm{PF}_{1}$ is obviously not reasonable. So the choice of processing objects should be adjusted according to the actual situation.

The selected objects in this paper are as follow: The mean instantaneous frequency of $\mathrm{PF}_{1}$ is $40 \mathrm{~Hz}$, the mean instantaneous frequency of $\mathrm{PF}_{2}$ is $120 \mathrm{~Hz}$ and the mean instantaneous frequency of $\mathrm{PF}_{3}$ is $260 \mathrm{~Hz}$. The results are shown in Figure 9.

The first three orders of PF components contain different frequencies. Although there are $40 \mathrm{~Hz}$ and $120 \mathrm{~Hz}$ frequency components in $\mathrm{PF}_{1}$, they have been greatly weakened. Therefore, MS can effectively weaken the mode mixing phenomenon of the simulation signal.

In order to verify the advantages of the proposed method VMD and EMD are introduced for comparison. Figure 10 is the EMD decomposition results of the simulation signal and Figure 11 is the VMD decomposition results of the simulation signal. From the results of EMD, the frequencies of the decomposed component are $40 \mathrm{~Hz}, 80 \mathrm{~Hz}$ and $160 \mathrm{~Hz}$ and $225 \mathrm{~Hz}$. The mode mixing of the EMD decomposition results is very serious and the three components can be obtained. Because the results of VMD are affected by the layer number of its decomposition, the results of decomposition are easily distorted. The simulation signal is composed of $40 \mathrm{~Hz}, 120 \mathrm{~Hz}$ and $260 \mathrm{~Hz}$. From the results of VMD, 
the frequencies of the decomposed component are $54 \mathrm{~Hz}, 130 \mathrm{~Hz}$ and $335 \mathrm{~Hz}$. Compared with the LMD-MS method proposed in this paper, it is obvious that the decomposition results in the paper are more accurate, which proves that the method proposed in this paper has strong advantages compared with other decomposition methods.
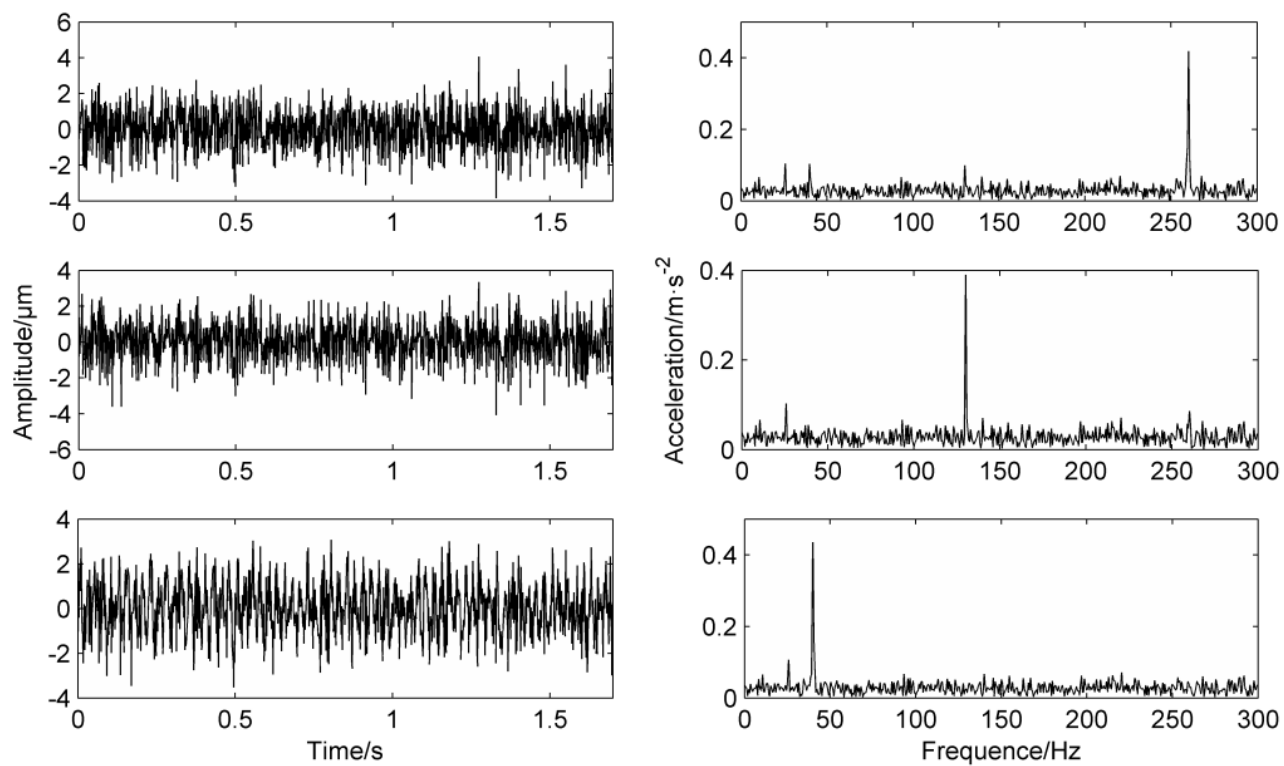

Figure 9. Simulation signal's decomposition results using LMD-MS.
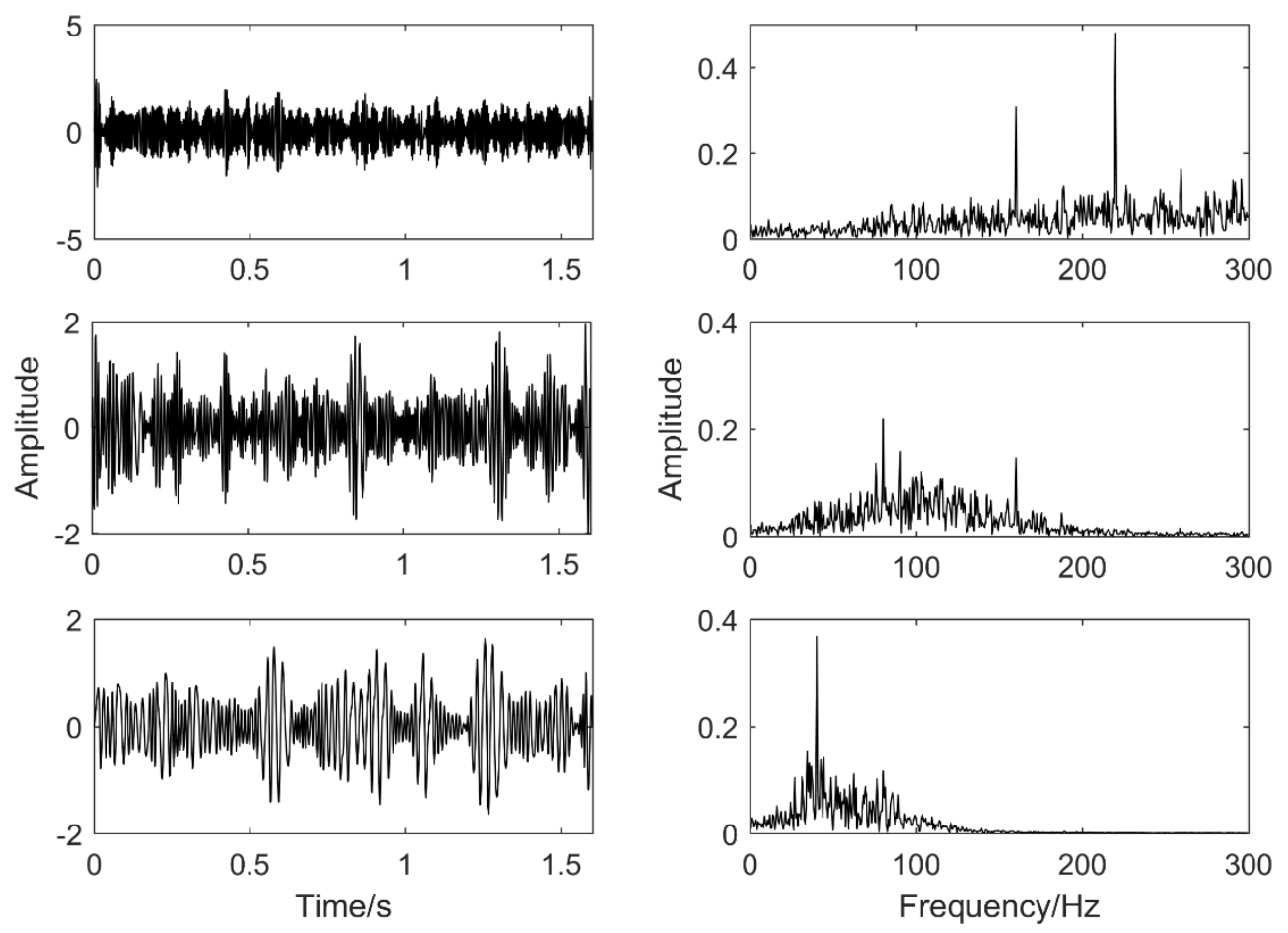

Figure 10. Simulation signal's decomposition results using EMD. 

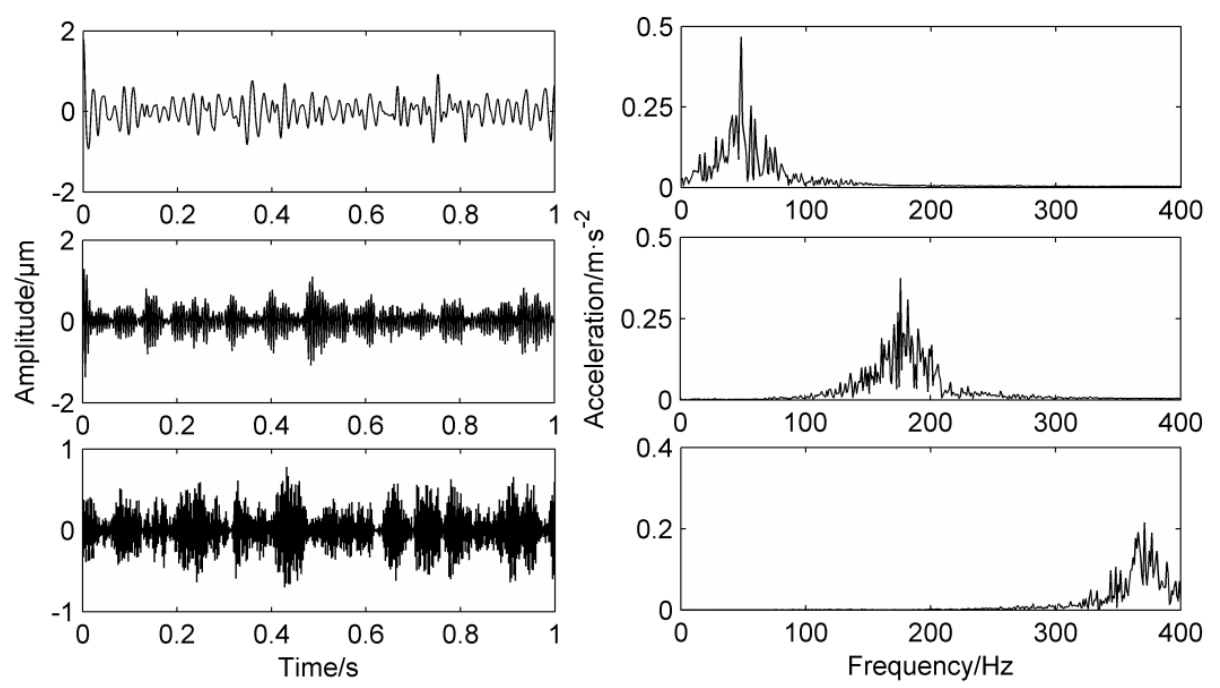

Figure 11. Simulation signal VMD decomposition results.

\section{LMD-MS Rolling Bearing Fault Signal Analysis}

In this paper, adopting Case Western Reserve University's fault bearing data for analysis [25], the speed is $1750 \mathrm{r} / \mathrm{min}$, the sampling frequency is $12,000 \mathrm{~Hz}$, and the basic frequency of the rotating shaft is $29.1 \mathrm{~Hz}$, the calculated inner ring fault frequency is about $157.9 \mathrm{~Hz}$, this paper selects 10,240 points of the data for analysis and research. Calculate the mean frequency energy's mean value of the original signal and filter the required frequency information. Take the frequency values before and after $5 \mathrm{~Hz}$ as the frequency energy mean length are shown in Figure 12. Figure 13 shows the time domain waveforms and envelope analysis results of the LMD decomposition of the fault signal. Take the first three layers PFs that are strongly related to the original signal.

In Figure 12, the mean values of the frequency energy at $29 \mathrm{~Hz}$ and $58 \mathrm{~Hz}$ are higher, and the mean value of the frequency energy at $160 \mathrm{~Hz}$ is the highest, thus the mask signal has been determined.

In the figure, there are higher peaks at the inner ring fault frequency of $158.3 \mathrm{~Hz}$ and its double frequency of $316.6 \mathrm{~Hz}$. Due to the fake frequency modulated by the signal during transmission process, there are higher peaks at the frequency of $29.1 \mathrm{~Hz}$ and the double frequency of $58.2 \mathrm{~Hz}$, while $98 \mathrm{~Hz}$ and $210 \mathrm{~Hz}$ have higher peaks. The results obtained by $\mathrm{PF}_{2}$ and $\mathrm{PF}_{3}$ are similar to $\mathrm{PF}_{1}$, which proves that the mode mixing phenomenon has occurred. PF1 is processed by the mask method and the result is shown in Figure 14. Obviously, the $29.1 \mathrm{~Hz}$ and the double frequency of $58.2 \mathrm{~Hz}$ are obviously weakened, which weakens the mode aliasing of LMD.

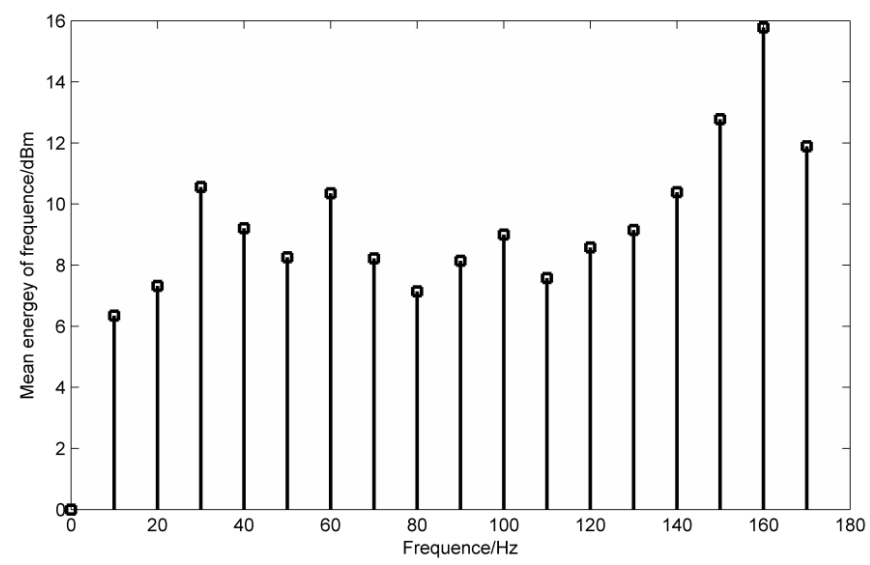

Figure 12. Mean energy distribution of the original signal frequency. 

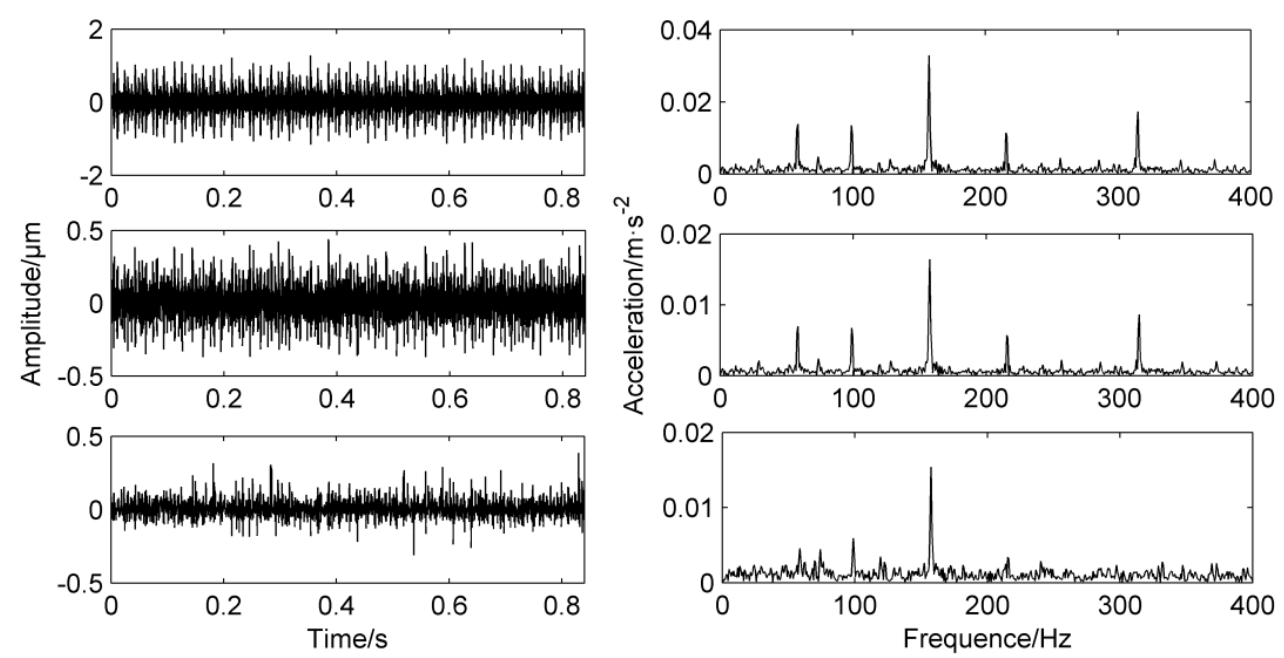

Figure 13. Original fault signal's decomposition results using LMD and envelope analysis.
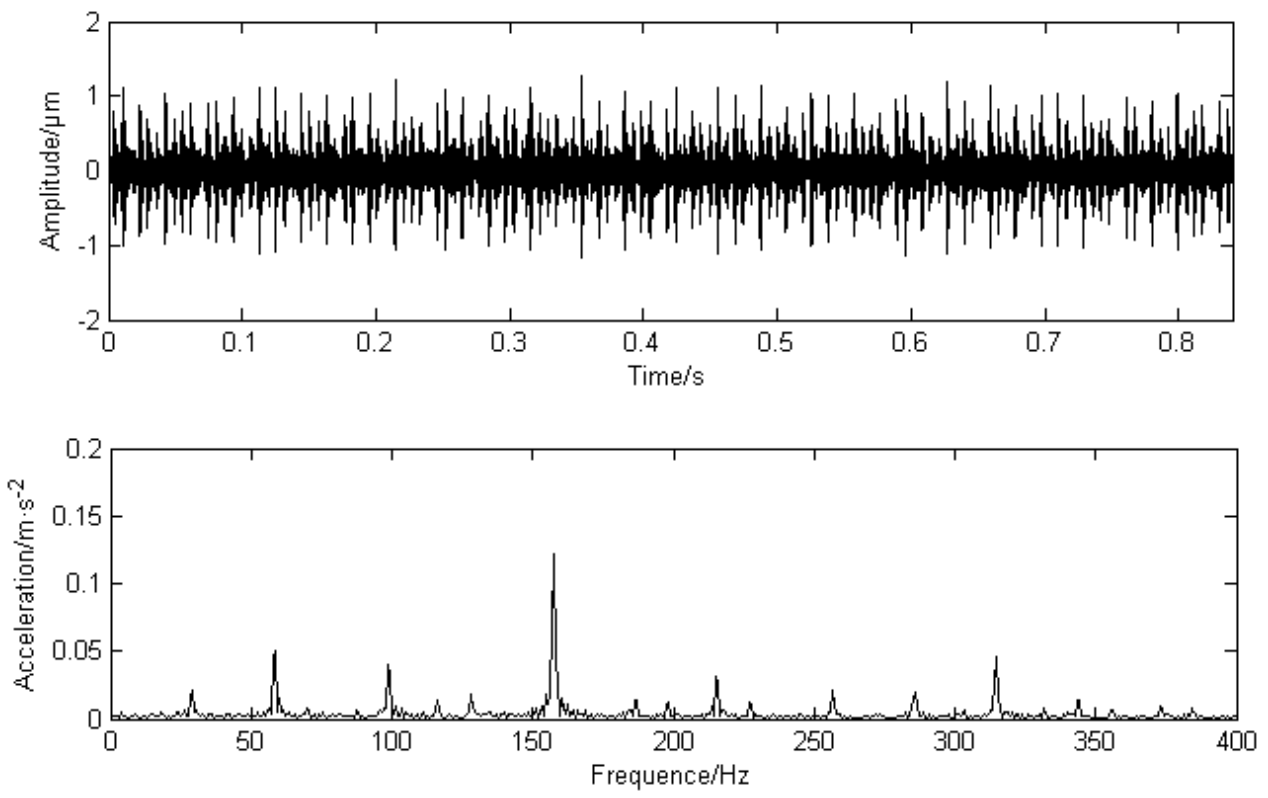

Figure 14. PF1 time-domain diagram and envelope analysis using MS.

From the comparison of the envelope analysis of the $\mathrm{PF}_{1}$ component after the mask processing, it can be seen that the peaks at $58 \mathrm{~Hz}$ and $100 \mathrm{~Hz}$ are greatly weakened, and higher peaks appear only at the inner fault frequency of $158.1 \mathrm{~Hz}$ and the double frequency of $316.2 \mathrm{~Hz}$. At the same time, the signal amplitude is reduced, which proves that MS has a certain noise reduction capability. After calculation, the correlation coefficient of $\mathrm{PF}_{1}$ is the highest, so $\mathrm{PF}_{1}$ is selected as the research object. In practical application, the component with the highest correlation coefficient can be selected as the research object.

Similar to the simulation signal, VMD is selected as the control method, and the number of selected decomposition layers is 3. The three components of VMD decomposition are shown in Figure 15, and the peak frequencies are at $50 \mathrm{~Hz}, 120 \mathrm{~Hz}$ and $355 \mathrm{~Hz}$ respectively. It is obvious that the frequency $157.9 \mathrm{~Hz}$ of the inner ring has not been decomposed, which is due to the nature of VMD itself. Therefore, it is proved that the method used in this paper is superior to other fault diagnosis methods. 

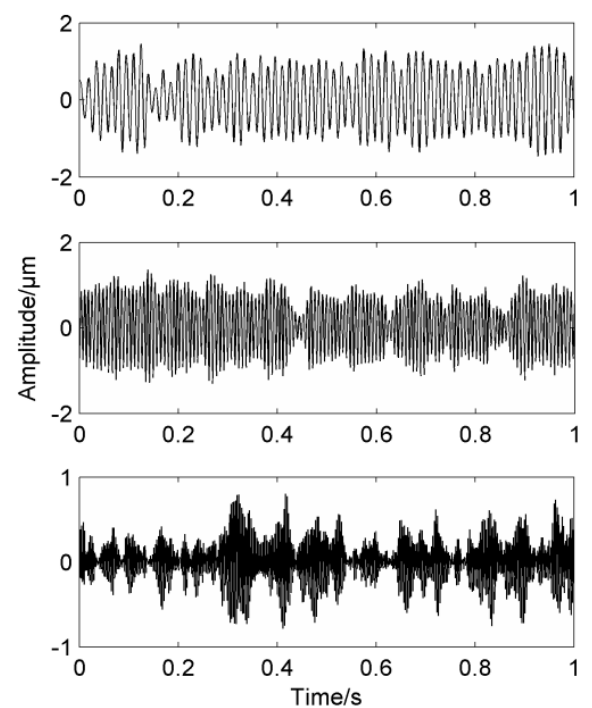
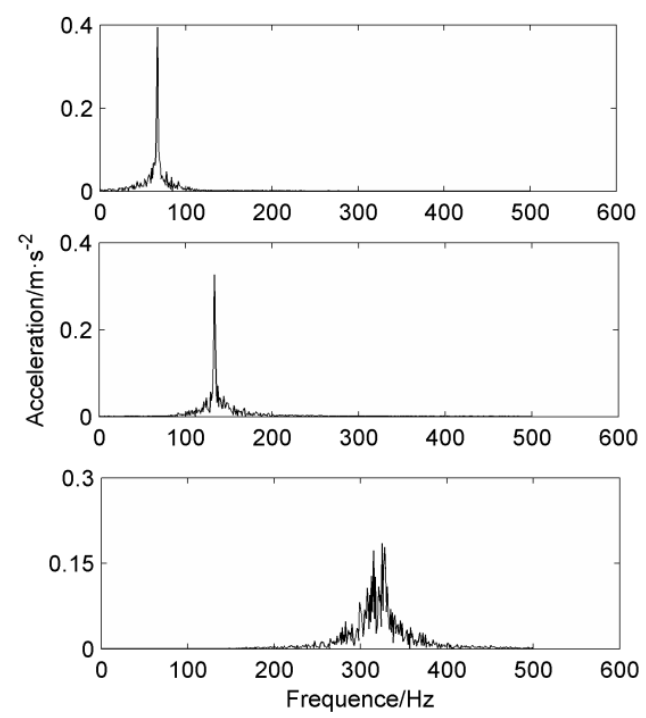

Figure 15. VMD decomposition of the measured signal.

Optimization degree of LMD-MS is studied from the kurtosis. The kurtosis values of $\mathrm{PF}_{1}, \mathrm{PF}_{2}$ and $\mathrm{PF}_{3}$ increased from 3.6, 3.3 and 3.2 to $28.4,27.6$ and 26.8, an increase of nearly eight times.

According to the signal-to-noise ratio formula:

$$
S N R=10 \lg \frac{2\left|X\left(k_{0}\right)\right|}{\sum_{k=0}^{L-1}|X(k)|^{2}-2\left|X\left(k_{0}\right)\right|^{2}}
$$

The $S, N$, and $L$ are respectively the signal energy, the noise energy, and the signal length, $X(k)$ and $X\left(k_{0}\right)$ are the peak at $k$ and the peak of the characteristic frequency in the spectrum. The signal-to-noise ratio of the signal at $157.9 \mathrm{~Hz}$ has increased by $19.1 \%$ before and after the use of the mask method.

\section{Conclusions}

(1) MS has a strong inhibitory effect on the mode mixing under strong noise background, and experiments show that MS has a certain ability to the noise reduction.

(2) LMD has a strong ability to decompose and analyze fault signals, but it will distort in strong noise background, and cause mode mixing and so on. It is difficult to get effective fault information.

(3) In this paper, MS is introduced into LMD, and a LMD-MS method which combines the mask signal method with LMD has been proposed. The signal is decomposed by LMD, and then the PF components are processed by MS to reduce the noise and alleviate the mode mixing. The simulation signal is used to verify the feasibility of the proposed method. The signal-to-noise ratio of the actual signal is increased by $19.1 \%$, and the weak fault features of the bearing are extracted successfully. It provides a new research idea for the weak fault feature extraction.

Author Contributions: L.Q. conceived and designed the experiments; Z.W. performed the experiments; L.Z. wrote the paper. All authors have read and approved the final manuscript.

Acknowledgments: This work was supported by the National Natural Science Foundation of China (Grant No. 51405327) and the Natural Science Foundation of Shanxi Province, China and Science, and Technology Foundation of Shanxi Provincial (No. 2015011063).

Conflicts of Interest: The authors declare no conflict of interest. 


\section{References}

1. Wang, Z.J.; Wang, J.Y. Weak Fault Diagnosis of Wind Turbine Gearboxes Based on MED-LMD. Entropy 2017, 19, 277. [CrossRef]

2. Wang, Z.J.; Wang, J.Y. A novel method for multi-fault feature extraction of a gear box under Strong background noise. Entropy 2018, 21, 10. [CrossRef]

3. Chen, G.; Hao, T.F.; Wang, H.F.; Zhao, B.; Wang, J.; Cheng, X.Y. Sensitivity Analysis and Experimental Research on Ball Bearing Early Fault Diagnosis Based on Testing Signal from Casing. J. Dyn. Syst. Meas. Cont. 2014, 136, 061009. [CrossRef]

4. Tian, Y.; Ma, J.; Lu, C.; Wang, Z. Rolling bearing fault diagnosis under variable conditions using LMD-SVD and extreme learning machine. Mech. Mach. Theory 2015, 90, 175-186. [CrossRef]

5. Wang, Z.; Han, Z.; Gu, F.; Gu, J.X.; Ning, S. A novel procedure for diagnosing multiple faults in rotating machinery. ISA Trans. 2015, 55, 208-218. [CrossRef] [PubMed]

6. Li, Z.; Chen, J.; Zi, Y.; Pan, J. Independence-oriented VMD to identify fault feature for wheel set bearing fault diagnosis of high speed locomotive. Mech. Syst. Signal Proc. 2017, 85, 512-529. [CrossRef]

7. Yuan, J.; Ji, F.; Gao, Y.; Zhu, J.; Wei, C.; Zhou, Y. Integrated ensemble noise-reconstructed empirical mode decomposition for mechanical fault detection. Mech. Syst. Signal Proc. 2018, 104, 323-346. [CrossRef]

8. Park, C.S.; Choi, Y.C.; Kim, Y.H. Early fault detection in automotive ball bearings using the minimum variance cepstrum. Mech. Syst. Signal Proc. 2013, 38, 534-548. [CrossRef]

9. Qiao, Z.; Lei, Y.; Lin, J.; Jia, F. An adaptive unsaturated bistable stochastic resonance method and its application in mechanical fault diagnosis. Mech. Syst. Signal Proc. 2017, 84, 731-746. [CrossRef]

10. Feng, Y.; Lu, B.; Zhang, D. Multiscale singular value manifold for rotating machinery fault diagnosis. J. Mech. Sci. Technol. 2117, 31, 99-109. [CrossRef]

11. Dragomiretskiy, K.; Zosso, D. Variational mode decomposition. IEEE Trans. Signal Proc. 2014, 62, 531-544. [CrossRef]

12. Wang, X.B.; Yang, Z.X.; Yan, X.A. Novel particle swarm optimization-based variational mode decomposition method for the fault diagnosis of complex rotating machinery. IEEE/ASME Trans. Mechatron. 2018, 23, 68-79. [CrossRef]

13. Yang, Z.X.; Zhong, J.H. A hybrid EEMD-based SampEn and SVD for acoustic signal processing and fault diagnosis. Entropy 2016, 18, 112. [CrossRef]

14. Smith, J.S. The local mean decomposition and its application to EEG perception data. J. R. Soc. Int. 2005, 2, 443. [CrossRef] [PubMed]

15. Li, Y.; Liang, X.; Yang, Y.; Xu, M.; Huang, W. Early Fault Diagnosis of Rotating Machinery by Combining Differential Rational Spline-Based LMD and K-L Divergence. IEEE Trans. Instrum. Meas. 2017, 66, 3077-3090. [CrossRef]

16. Wang, L.; Liu, Z.; Miao, Q.; Zhang, X. Time-frequency analysis based on ensemble local mean decomposition and fast kurtogram for rotating machinery fault diagnosis. Mech. Syst. Signal Proc. 2018, 103, 60-75. [CrossRef]

17. Deng, W.; Yao, R.; Sun, M.; Zhao, H.; Luo, Y.; Dong, C. Study on a novel fault diagnosis method based on integrating EMD, fuzzy entropy, improved PSO and SVM. J. Vibroeng. 2017, 19, 2562-2577.

18. Lu, C.; Wang, Z.Y.; Qin, W.L.; Ma, J. Fault diagnosis of rotary machinery components using a stacked denoising autoencoder-based health state identification. Signal Proc. 2017, 130, 377-388. [CrossRef]

19. Gao, Y.; Villecco, F.; Li, M.; Song, W. Multi-Scale permutation entropy based on improved LMD and HMM for rolling bearing diagnosis. Entropy 2017, 19, 176. [CrossRef]

20. Ling, X.; Yan, X. Comparative study on the performance of LMD method and EMD method in steam turbine rotor fault diagnosis. J. Chin. Soc. Power Eng. 2014, 34, 945-951.

21. Xu, T.; Yin, Z.; Cai, D.; Zheng, D. Fault diagnosis for rotating machinery based on Local Mean Decomposition morphology filtering and Least Square Support Vector Machine. J. Intell. Fuzzy Syst. 2017, 32, 2061-2070. [CrossRef]

22. Rilling, G.; Flandrin, P.; Goncalves, P. Empirical mode decomposition, fractional Gaussian noise and Hurst exponent estimation. In Proceedings of the IEEE International Conference on Acoustics, Speech, and Signal Processing, Philadelphia, PA, USA, 23 March 2005. 
23. Deering, R.; Kaiser, J.F. The use of a masking signal to improve empirical mode decomposition. In Proceedings of the IEEE International Conference on Acoustics, Speech, and Signal Processing, Philadelphia, PA, USA, 23 March 2005.

24. Nakayama, J.; Kanno, K.; Uchida, A. Laser dynamical reservoir computing with consistency: An approach of a chaos mask signal. Opt. Express 2016, 24, 8679-8692. [CrossRef] [PubMed]

25. Case Western Reserve University. Case Western Reserve University Bearing Data Center. Available online: https:/ / csegroups.case.edu/bearingdatacenter/pages/download-data-file (accessed on 1 October 2009).

2018 by the authors. Licensee MDPI, Basel, Switzerland. This article is an open access article distributed under the terms and conditions of the Creative Commons Attribution (CC BY) license (http:/ / creativecommons.org/licenses/by/4.0/). 